\title{
Monitoring of Urban Ambient Air Quality at Locations of Preschool and School Facilities in Serbia
}

\author{
BRANISLAVA I. MATIĆ, Institute of Public Health of Serbia \\ „Dr Milan Jovanović Batut", Belgrade \\ Original scientific paper \\ SNEŽANA M. DEJANOVIĆ, Institute of Public Health of Serbia \\ UDC: 502.3:502.175(497.11) \\ 502.3:504.5(497.11) \\ „Dr Milan Jovanović Batut“, Belgrade \\ DOI: 10.5937/tehnika2105673M \\ DRAGANA D. JOVANOVIĆ, Institute of Public Health of Serbia
}

„Dr Milan Jovanović Batut“, Belgrade

Exposing children to environmental pollutants during periods significant for their physiological development can lead to long-lasting health effects, dysfunction, and disease. The aim of this paper was to focus on a relatively scarce ambient AQ monitoring practice at the locations of preschol and school institutions in Serbia, within the local network of $A Q$ monitoring stations. This was a cross-sectional study covering period 2015 to 2020. Measuring points $\left(\mathrm{SO}_{2}, \mathrm{NO}_{2}\right.$, soot, PM 10 ) were in front of kindergardens, primary and secondary schools in urban settlements. Results originate from the continuous public health programme conducted by the Institute of Public Health of Serbia. Only PM $M_{10}$ and $B(a) P$ values at some points have exceeded annual limit values in front of chosen facilities in 2020. In sum, both the absolute number of urban ambient AQ measuring points monitored by the local network of Institutes of Public Health and those in front of the educational facilities have increased in this period: from 73/17 to 172/26. In a percentual view, these facilities are chosen more seldom within this time frame, dropping from 23.29 to $15.12 \%$ of all AQ monitoring points. As such facilities are mainly located at important city intersections, in order to assess the exposure to air pollution of vulnerable population groups (children from preschool to adolescence age), there is a need to locate measuring points near them.

Key words: educational facilities, ambient air pollution, measuring points

\section{INTRODUCTION}

According to their location, schools in the administrative area of large cities can be positioned in the central urban core of the city, in the broader urban area and in rural suburbs, which determines the potential risks from the environment [1]. Given the length of time children spend in school, the school environment represents a significant microclimatic environment, in terms of the impact of air pollution on their respiratory health, above all. According to International Review of Curriculum and Assessment Frameworks Internet Archive children in developed countries spend 175 to 220 days a year in school, five to eight hours a day [2].

Author's address: Branislava Matić, Institute of $\mathrm{Pu}$ blic Health of Serbia „Dr Milan Jovanović Batut“, Belgrade, Dr Subotića Starijeg 5

e-mail: brankicam@batut.org.rs

Paper received: 20.08.2021.

Paper accepted: 22.09.2021.
It is not a negligible fact that schools in urban areas are often located near busy roads, which causes incre-ased exposure of children to pollutants, since children attend classes during the day, every day during the working week, which coincides with the maximum traffic flux [3]. If we take $\mathrm{PM}_{10}$ particles as an example of traffic induced pollutants, their transport from the ambient air of the school environment to the indoor air of classrooms has been proven, as well as harmful cosequences for students' respiratory health [4].

According to other studies, a link has been recognized between decreased lung function, inflammatory processes of the respiratory tract and asthma, in children who live and attend school near busy roads [5; $6]$. The other two pollutants, which, if measured in indoor air, represent indicators of traffic intensity in the streets near the examined school, are nitrogen dioxide and carbon monoxide [7;8].

Schools located in rural settlements represent a different type of air pollution source, and thus a risk to 
the respiratory health of students. First of all, unlike traffic air pollution, where mobile sources of pollution (motor vehicle exhaust) are involved, schools in rural areas are stationary sources of air pollution. Namely, they are most often not connected to the centralized system of heat energy supply, but they mainly use heating bodies on solid fossil fuels (coal, wood) to generate heat. In the case of this type of heating of the school space, children are exposed to polluted air inside the classrooms, as well as in the yard, due to the emission of coal / wood combustion products into the ambient air [9].

The use of solid fossil fuels for heating, in terms of energy, is at the bottom of the scale in terms of combustion efficiency and cleanliness [10]. Smoke from the combustion of solid fossil fuels produces a large number of hazardous pollutants, including respirable particles, carbon monoxide (CO), nitrogen oxides, formaldehyde, benzene, 1,3 butadiene, polycyclic aromatic hydrocarbons (PAHs), like benzo[a]pyrene, along with many other toxic compounds. In underdeveloped areas, a large part of households, including schools, most often use this type of fuel for cooking and heating, so the concentrations of the listed pollutants are highest in indoor air [11]. They typically burn in energy-inefficient radiators, which combined with poor room ventilation habits can generate dense smoke in such rooms. It is important to note that children are exposed to these pollutants only during their stay in school, because after their departure, the energy source is turned off, so they are much more exposed in the classrooms themselves $[10 ; 12]$.

The aim of this paper was to focus on a relatively scarce ambient AQ monitoring practice at the locations of preschol and school institutions in Serbia, within the local network of AQ monitoring stations. The specific objective was to analyze the quantitative values of air quality monitoring, in order to assess the possible need to improve the monitoring process, in terms of increasing the number of sites or adding some parameters at defined measuring points.

\section{METHODS AND DATA COLLECTION}

The research tool used in this cross-sectional study is a data base formed and regularly updated by the Table 1. GIS coordinates of educational facilities
Institute of Public Health of Serbia (IPHS), as a segment of a continuous AQ monitoring process implemented for the Ministry of Health, through regular reporting of local IPHs from the Network. Sampling, measuring and analyzing level of defined indicators are done by environmental health departments of local / regional IPHs. Data collation, processing and analysis are undertaken by the IPHS, whose assignment is to forward its remarks on possible public health risks to decision-makers. Due to the clear market conditions established in the field of operation of accredited laboratories, the data collected at the IPHS may vary from year to year [4].

Indicators that are monitored are grouped according to the nature of pollution sources, population coverage, traffic density and potential public health risks are: $\mathrm{SO}_{2}$, soot, $\mathrm{NO}_{2}$ and other specific pollutants, such as $\mathrm{NH}_{3}, \mathrm{PM}_{10}$, heavy metals, and traffic-originated organic chemicals). Together with the key stakeholders at the local level (local self-government, local IPH), each municipality/town produces a Local Action Plan for AQ monitoring, defining the time schedule of monitoring activities and spatial distribution of sampling points, sources of air pollution and choice of pollutants in need of monitoring. Sulfur dioxide and BC are measured daily due to the strong correlation among their ambient air concentrations and fossil fuel combustion, as Serbia's key energy source is lignite coal [13]. $\mathrm{NO}_{2}$ is the most frequently measured specific pollutant, on daily basis. Presence of particulate pollution in ambient air is monitored through sampling of coarse particles of total atmospheric deposition $\left(\mathrm{mg} / \mathrm{m}^{2} /\right.$ day $)$, $\mathrm{PM}_{10}$ and $\mathrm{PM}_{2.5}$, with different measurement frequency, according to local AQ monitoring plans and specific indication, depending on pollution sources and financial resources of the local administration and the polluter, per se [14]. Mapping school locations was done using GIS.

\section{RESULTS}

Table 1 shows precise GIS location of all measuring points (PS -primary schools, KG-kindergartens and one secondary medical school, Sec.school; SOS Childrens Village) together with the sellected pollutants monitored at each site.

\begin{tabular}{|c|c|c|c|}
\hline \multicolumn{2}{|l|}{ Facilities (27) } & GIS & Pollutants measured \\
\hline Rakovica, Beograd & PS „Nikola Tesla” & $\begin{array}{l}44^{\circ} 44^{\prime} 47.55^{\prime \prime} \mathrm{N} \\
20^{\circ} 26^{\prime} 21.56^{\prime \prime} \mathrm{E}\end{array}$ & $\begin{array}{l}\mathrm{SO}_{2} \text {, soot, } \mathrm{NO}_{2} \text {, indicative: } \mathrm{PM}_{10} \text { with heavy } \\
\text { metals analysis }(\mathrm{As}, \mathrm{Cd}, \mathrm{Ni}, \mathrm{Pb}), \mathrm{B}(\mathrm{a}) \mathrm{P}\end{array}$ \\
\hline Višnjica, Beograd & PS "Milena Pavlović Barili" & $\begin{array}{l}44^{\circ} 49^{\prime} 04.88^{\prime \prime} \mathrm{N} \\
20^{\circ} 32^{\prime} 04.86^{\prime \prime} \mathrm{E} \\
\end{array}$ & $\mathrm{SO}_{2}$, soot, $\mathrm{NO}_{2}$, benzene \\
\hline Valjevo & PS "Sestre Ilić" & $\begin{array}{l}44^{\circ} 16^{\prime} 09.59^{\prime \prime} \mathrm{N} \\
19^{\circ} 52^{\prime} 53.01^{\prime \prime} \mathrm{E}\end{array}$ & $\mathrm{SO}_{2}$, soot, $\mathrm{NO}_{2}$ \\
\hline
\end{tabular}




\begin{tabular}{|c|c|c|c|}
\hline \multicolumn{2}{|l|}{ Facilities (27) } & \multirow{2}{*}{$\begin{array}{l}\text { GIS } \\
\begin{array}{l}42^{\circ} 32^{\prime} 01.88^{\prime \prime} \mathrm{N} \\
21^{\circ} 53^{\prime} 53.90^{\prime \prime} \mathrm{E}\end{array}\end{array}$} & \multirow{2}{*}{$\begin{array}{l}\text { Pollutants measured } \\
\mathrm{SO}_{2}, \text { soot, } \mathrm{NO}_{2}, \mathrm{AD}\end{array}$} \\
\hline Vranje & PS „Svetozar Marković” & & \\
\hline Zvečan & PS „,Vuk Karadžić” & $\begin{array}{l}42^{\circ} 54^{\prime} 38.81^{\prime \prime} \mathrm{N} \\
20^{\circ} 50^{\prime} 19.10^{\prime \prime} \mathrm{E}\end{array}$ & $\mathrm{SO}_{2}$, soot, $\mathrm{NO}_{2}$ \\
\hline Kosjerić & PS “Mito Igumanović”" & $\begin{array}{l}43^{\circ} 59^{\prime} 40.50^{\prime \prime} \mathrm{N} \\
19^{\circ} 54^{\prime} 25.85^{\prime \prime} \mathrm{E}\end{array}$ & $A D$ \\
\hline Kragujevac & PS „M. Jovanović” & $\begin{array}{l}44^{\circ} 01^{\prime} 48,66^{\prime \prime} \mathrm{N} \\
20^{\circ} 54^{\prime} 20,54^{\prime \prime} \mathrm{E}\end{array}$ & $\begin{array}{l}\mathrm{SO}_{2}, \text { soot }, \mathrm{NO}_{2}, \mathrm{PM}_{10} \text { with heavy metals analysis } \\
(\mathrm{Pb}, \mathrm{Cd}, \mathrm{As}, \mathrm{Ni})\end{array}$ \\
\hline \multirow{3}{*}{ Niš } & „D. Radović“, Medijana, PS & $\begin{array}{l}43^{\circ} 19^{\prime} 02,81^{\prime \prime} \mathrm{N} \\
21^{\circ} 53^{\prime} 09,86^{\prime \prime} \mathrm{E}\end{array}$ & \multirow{2}{*}{$\mathrm{SO}_{2}$, soot, $\mathrm{NO}_{2}$} \\
\hline & PS, „Čegar” Pantelej & $\begin{array}{l}43^{\circ} 19^{\prime} 57,72^{\prime \prime} \mathrm{N} \\
21^{\circ} 55^{\prime} 43,02^{\prime \prime} \mathrm{E}\end{array}$ & \\
\hline & PS ,St Sava” & $\begin{array}{l}43^{\circ} 19^{\prime} 18,89^{\prime \prime} \mathrm{N} \\
21^{\circ} 55^{\prime} 11,07^{\prime \prime} \mathrm{E}\end{array}$ & PMIo with heavy metals analysis $(\mathrm{Pb}, \mathrm{Cd}, \mathrm{As}, \mathrm{Ni})$ \\
\hline Kać & PS „Đura Jakšić““, & $\begin{array}{l}45^{\circ} 17^{\prime} 59.45^{\prime \prime} \mathrm{N} \\
19^{\circ} 56^{\prime} 26.56^{\prime \prime} \mathrm{E}\end{array}$ & $\begin{array}{l}\mathrm{SO}_{2}, \mathrm{NO}_{2}, \mathrm{PM}_{10} \text { with heavy metals analysis }(\mathrm{Pb} \text {, } \\
\mathrm{Cd}, \mathrm{Ni}, \mathrm{As}), \mathrm{B}(\mathrm{a}) \mathrm{P}, \mathrm{PM}_{2,5}\end{array}$ \\
\hline Požarevac & PS „Kralj Aleksandar I” & $\begin{array}{l}44^{\circ} 36^{\prime} 52.2^{\prime \prime} \mathrm{N} \\
21^{\circ} 10^{\prime} 49.9^{\prime \prime} \mathrm{E}\end{array}$ & $\begin{array}{l}\mathrm{SO}_{2}, \partial u m, \mathrm{NO}_{2}, \mathrm{AD} \text { with heavy metals analysis } \\
(\mathrm{Pb}, \mathrm{Cd}, \mathrm{Zn})\end{array}$ \\
\hline Vranovo & PS “Dositej Obradović” & $\begin{array}{l}44^{\circ} 39^{\prime} 50.55^{\prime \prime} \mathrm{N} \\
20^{\circ} 55^{\prime} 27.08^{\prime \prime} \mathrm{E}\end{array}$ & $\begin{array}{l}P M_{10} \text { with heavy metals analysis }(\mathrm{Pb}, \mathrm{Cd}, \mathrm{Ni}, \mathrm{As}) \text {, } \\
B(a) P\end{array}$ \\
\hline Subotica & "S. Marinković" Mala škola & $\begin{array}{l}46^{\circ} 05^{\prime} 34^{\prime \prime} \mathrm{N} \\
19^{\circ} 40^{\prime} 20^{\prime \prime} \mathrm{E}\end{array}$ & $\begin{array}{l}\text { PM } 10 \text { with heavy metals analysis }(\mathrm{Pb}, \mathrm{Cd}, \mathrm{Ni}, \mathrm{As}) \text {, } \\
\mathrm{PM}_{2,5}\end{array}$ \\
\hline \multirow{4}{*}{ Valjevo } & $\mathrm{KG}$, ,Bubamara“ & $\begin{array}{l}44^{\circ} 16^{\prime} 33.64^{\prime \prime} \mathrm{N} \\
19^{\circ} 52^{\prime} 24.96^{\prime \prime} \mathrm{E}\end{array}$ & \multirow{4}{*}{$\mathrm{SO}_{2}$, soot, $\mathrm{NO}_{2}$} \\
\hline & KG „Pčelica” & $\begin{array}{l}44^{\circ} 17^{\prime} 01.21^{\prime \prime} \mathrm{N} \\
19^{\circ} 52^{\prime} 21.99^{\prime \prime} \mathrm{E}\end{array}$ & \\
\hline & KG „Kolibri” & $\begin{array}{l}44^{\circ} 16^{\prime} 56^{\prime \prime} \mathrm{N} \\
19^{\circ} 55^{\prime} 3^{\prime \prime} \mathrm{E} \\
\end{array}$ & \\
\hline & KG „Vidra“ & $\begin{array}{l}44^{\circ} 15^{\prime} 54.16^{\prime \prime} \mathrm{N} \\
19^{\circ} 53^{\prime} 49.96^{\prime \prime} \mathrm{E}\end{array}$ & \\
\hline Zvečan & \multirow{4}{*}{ Kindergartens } & $\begin{array}{l}42^{\circ} 54^{\prime} 39.92^{\prime \prime} \mathrm{N} \\
20^{\circ} 50^{\prime} 12,94^{\prime \prime} \mathrm{E}\end{array}$ & \multirow{4}{*}{ atmospheric deposition $(A D)$} \\
\hline Leposavić & & $\begin{array}{l}43^{\circ} 06^{\prime} 16.98^{\prime \prime} \mathrm{N} \\
20^{\circ} 48^{\prime} 08.91^{\prime \prime} \mathrm{E}\end{array}$ & \\
\hline Lešak & & $\begin{array}{l}43^{\circ} 10^{\prime} 09.83^{\prime \prime} \mathrm{N} \\
20^{\circ} 44^{\prime} 23.68^{\prime \prime} \mathrm{E}\end{array}$ & \\
\hline Zubin Potok & & $\begin{array}{l}42^{\circ} 55^{\prime} 24.72^{\prime \prime} \mathrm{N} \\
20^{\circ} 40^{\prime} 22.58^{\prime \prime} \mathrm{E}\end{array}$ & \\
\hline Kragujevac & KG Ilićevo, „Čuperak” & $\begin{array}{l}44^{\circ} 01^{\prime} 48,22^{\prime \prime} \mathrm{N} \\
20^{\circ} 57^{\prime} 06,44^{\prime \prime} \mathrm{E}\end{array}$ & $\mathrm{SO}_{2}$, soot, $\mathrm{NO}_{2}$ \\
\hline Niš & KG Pantelej, „Bubamara” & $\begin{array}{l}43^{\circ} 19^{\prime} 43,00^{\prime \prime} \mathrm{N} \\
21^{\circ} 54^{\prime} 22,13^{\prime \prime} \mathrm{E}\end{array}$ & $\begin{array}{l}\text { AD with heavy metals analysis }(\mathrm{Pb}, \mathrm{Cd}, \mathrm{Ni}, \mathrm{Cr} \\
\mathrm{SO}_{2}, \mathrm{NO}_{2} \text { (traffic-related) }\end{array}$ \\
\hline Niška Banja & KG „Pahuljica” & $\begin{array}{l}43^{\circ} 29^{\prime} 81.62^{\prime \prime} \mathrm{N} \\
21^{\circ} 98^{\prime} 48.30^{\prime \prime} \mathrm{E}\end{array}$ & $A D$ with heavy metals analysis $(\mathrm{Pb}, \mathrm{Cd}, \mathrm{Ni}, \mathrm{Cr})$ \\
\hline Sevojno & KG ,Maslačak“ & $\begin{array}{l}43^{\circ} 50^{\prime} 40.83^{\prime \prime} \mathrm{N} \\
19^{\circ} 53^{\prime} 45.09^{\prime \prime} \mathrm{E}\end{array}$ & $\begin{array}{l}\text { AD with heavy metals analysis }(\mathrm{Pb}, \mathrm{Cd}, \mathrm{Zn}, \mathrm{Ni} \text {, } \\
\mathrm{As}, \mathrm{Cu})\end{array}$ \\
\hline Kragujevac & Medical second.school & $\begin{array}{l}44^{\circ} 01^{\prime} 11,79^{\prime \prime} \mathrm{N} \\
20^{\circ} 54^{\prime} 33,36^{\prime \prime} \mathrm{E}\end{array}$ & $A D$ with heavy metals analysis $(P b, C d, Z n)$ \\
\hline Sremska Kamenica & $\begin{array}{l}\text { SOS Ch.Village „Dr Milorad } \\
\text { Pavlović“" }\end{array}$ & $\begin{array}{l}45^{\circ} 13^{\prime} 31.41^{\prime \prime} \mathrm{N} \\
19^{\circ} 50^{\prime} 42.69^{\prime \prime} \mathrm{E}\end{array}$ & $\begin{array}{l}\mathrm{SO}_{2}, \mathrm{NO}_{2}, \mathrm{NO}, \mathrm{NO}_{X}, \mathrm{CO}, \mathrm{O}_{3}, \mathrm{BeToXy}, \mathrm{PM}_{10} \text { with } \\
\text { heavy metals analysis }(\mathrm{Pb}, \mathrm{Cd}, \mathrm{Ni}, \mathrm{As}), \mathrm{B}(\mathrm{a}) \mathrm{P} \text {, } \\
\mathrm{PM}_{2,5} \text {, }\end{array}$ \\
\hline
\end{tabular}


The exception among these educational institutions is the SOS Children's Village (Sremska Kamenica), as the site is not only a teaching, but also a residential space, where complete care is taken of children without parental care in an organized and humane way. The fact that children are going through all of their daily routine at the same spot could be the reason why at this site the most number and type of pollutants are monitored simultaneously.

Although the total number of measuring points in the Local AQ monitoring network in the period 20152020 is, actually, increasing, the number of educational facilities used as sampling points is in decline (Figures 1\&2.).

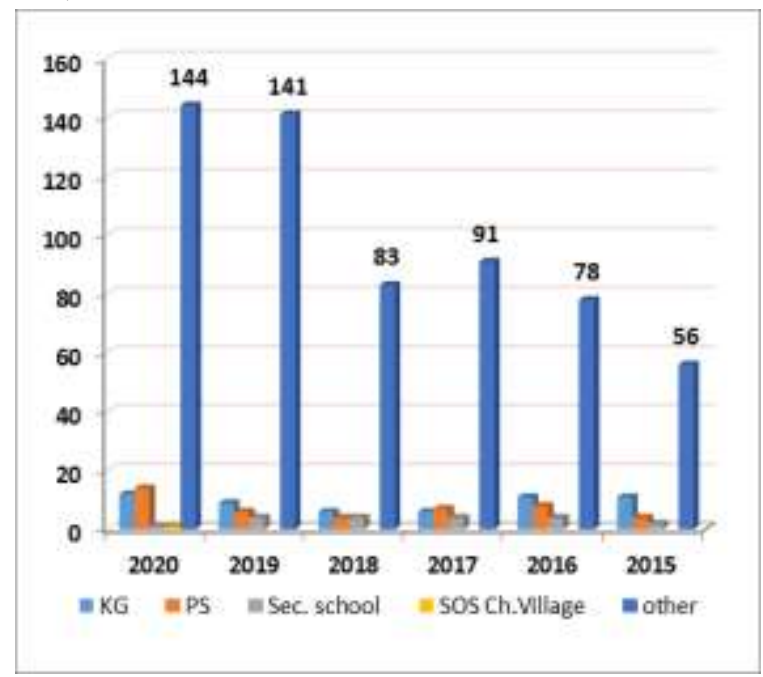

Figure 1 - Measuring points in front of educational facilities

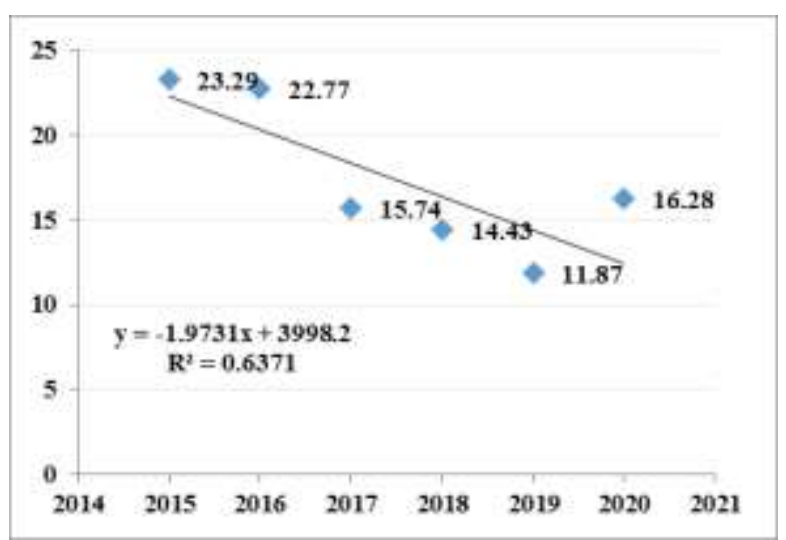

Figure 2 - Measuring points in front of educational facilities - The declining trend of coverage (2015-2021)

\subsection{Concentration of specific pollutants in 2020}

As shown in the three following tables, pollutants measured in 2020 in front of educational facilities were distributed in the following frequency: $\mathrm{SO}_{2} / 13$, soot/13, $\mathrm{NO}_{2} / 15$ atmospheric deposition/12 and $\mathrm{PM}_{10} / 5$. Analyzing values of $\mathrm{SO}_{2}, \mathrm{NO}_{2}$ and soot, being measured daily, no exceedances of the annual limit value were recorded for any of these parameters at the measuring points close to educational facilities (Table 2).

Table 2. $\mathrm{SO}_{2}$, soot, $\mathrm{NO}_{2}$ (measured on daily basis, $\mu \mathrm{g} / \mathrm{m}^{3}$ )

\begin{tabular}{|c|c|c|c|}
\hline Parameters & $\mathrm{SO}_{2}$ & Soot & $\mathrm{NO}_{2}$ \\
\hline Educational facilities & \multicolumn{3}{|c|}{$\left(\mu \mathrm{g} / \mathrm{m}^{3}\right)$} \\
\hline \multicolumn{4}{|c|}{ Primary schools } \\
\hline „Nikola Tesla“, Rakovica & $<10$ & 14.33 & 22.67 \\
\hline $\begin{array}{l}\text { „Milena Pavlović Barili“, } \\
\text { Višnjica }\end{array}$ & $<10$ & 11.63 & 12.82 \\
\hline „Sestre Ilić“", Valjevo & 23.84 & 11.91 & 18.61 \\
\hline $\begin{array}{l}\text { „Svetozar Marković”, } \\
\text { Vranje }\end{array}$ & 36.39 & 10.84 & 15.13 \\
\hline „Vuk Karadzic“, Zvečan & 12.29 & 8.76 & 5.34 \\
\hline $\begin{array}{ll}\text { „M. Jovanović“ } \\
\text { Kragujevac }\end{array}$ & 5.42 & 8.20 & 13.83 \\
\hline „Čegar”, Pantelej & & 12.53 & \\
\hline „Đura Jakšić“", Kać & 6.45 & & 16 \\
\hline $\begin{array}{l}\text { „Kralj Aleksandar I“ } \\
\text { Požarevac }\end{array}$ & 20.25 & 9.65 & 29.11 \\
\hline \multicolumn{4}{|c|}{ Kindergartens } \\
\hline „Pčelica” Valjevo & 25.43 & 14.39 & 20.57 \\
\hline „Kolibri”, Novo Valjevo & 29.77 & 16.71 & 20.62 \\
\hline „Vidra“, Valjevo & 31.58 & 9.99 & 21.80 \\
\hline $\begin{array}{l}\text { „Bubamara“, Podgorska } \\
\text { bb, Valjevo }\end{array}$ & 27.67 & 8.76 & 15.63 \\
\hline „Bubamara“, Pantelej, Niš & 10.65 & & 9.16 \\
\hline \multicolumn{4}{|c|}{ Other } \\
\hline $\begin{array}{l}\text { Secondary Medical School, } \\
\text { Kragujevac }\end{array}$ & & 18.50 & 27.25 \\
\hline $\begin{array}{l}\text { SOS Children's village „Dr } \\
\text { Milorad Pavlović“ }\end{array}$ & 8.41 & & 17.16 \\
\hline $\begin{array}{l}\begin{array}{l}\text { Annual } \\
\left(\mu \mathrm{g} / \mathbf{m}^{3}\right)\end{array} \\
\end{array}$ & & & \\
\hline
\end{tabular}

Atmospheric deposition in front of educational institutions was monitored at 11 out of 72 measuring points in 2020, which is slightly more than $15 \%$ $(15.3 \%)$ of all measuring points at 9 out of 37 urban settlements within the local AQ network (Table 3).

Only at one point, in Kragujevac, the mean annual concentration has exceeded the annual limit value of $200 \mathrm{mg} / \mathrm{m}^{2} /$ day. At neither of the points mean annual values for heavy metals in atmospheric deposition $(\mathrm{Pb}$, $\mathrm{Cd}, \mathrm{Zn}, \mathrm{Ni}$ ) were of any significance.

Less than 3\% (2.9) of all measuring points in the survey had monitoring of $\mathrm{PM}_{10}$ particles in front of educational facilities, together with heavy metals contents (Table 4). In front of 2 out of 5 schools, $\mathrm{PM}_{10}$ concentration values were higher than the average 
anual limit value of $40 \mu \mathrm{g} / \mathrm{m}^{3}$. As for the adherent specific pollutants, only values for $\mathrm{B}(\mathrm{a}) \mathrm{P}$ at all 3 points had mean ALV beyound the reccomened $1.0 \mathrm{ng} / \mathrm{m}^{3}$.

Table 3. Atmospheric deposition with heavy metals in 2020

\begin{tabular}{|c|c|c|c|c|c|c|}
\hline Parameters & AD & $\mathbf{P b}$ & Cd & $\mathrm{Zn}$ & $\mathrm{Ni}$ & $\mathrm{Cr}$ \\
\hline $\begin{array}{l}\text { Educational } \\
\text { facilities }\end{array}$ & $\begin{array}{l}\mathrm{mg} / \mathrm{m}^{2} / \\
\text { day }\end{array}$ & \multicolumn{5}{|c|}{$\mu \mathrm{g} / \mathrm{m}^{2} /$ day } \\
\hline $\begin{array}{l}\text { P.S. „Svetozar } \\
\text { Marković”,Vra } \\
\text { nje }\end{array}$ & 58.41 & & & & & \\
\hline $\begin{array}{l}\text { KG } \\
\text { K.Mitrovica }\end{array}$ & 110.57 & 13.8 & $<1$ & 193 & $<7$ & $<4$ \\
\hline KG Zvečan & 107.86 & 10.2 & $<1$ & 83.2 & $<7$ & $<4$ \\
\hline KG, Leposavić & 105.06 & 8.3 & $<1$ & 33.2 & $<7$ & $<4$ \\
\hline KG, Lešak & 138.35 & 14.5 & $<1$ & 18.2 & $<7$ & $<4$ \\
\hline \begin{tabular}{|l|} 
KG Zubin \\
Potok
\end{tabular} & 168.81 & 8.7 & $<1$ & 49.6 & $<7$ & $<4$ \\
\hline P.S. Kosjerić & 94.77 & 1.9 & 0.1 & 29.1 & 6.3 & \\
\hline $\begin{array}{l}\text { Second.me- } \\
\text { dical school, } \\
\text { Kragujevac }\end{array}$ & 274.26 & 12.1 & 0.05 & 345 & $<1$ & \\
\hline $\begin{array}{l}\text { KG Pantelej, } \\
\text {,Bubamara" }\end{array}$ & 144.83 & 6.2 & 1.5 & 13.6 & 2,1 & \\
\hline \begin{tabular}{|l|} 
KG „Pahuljica” \\
Niška Banja \\
\end{tabular} & 209.33 & 5.5 & 1.6 & 16.3 & 6.2 & \\
\hline \multirow[t]{2}{*}{$\begin{array}{l}\text {,Kralj } \\
\text { Aleksandar I”, } \\
\text { Požarevac P.S. } \\
\end{array}$} & 106.7 & 5.2 & 0.21 & 12.0 & & \\
\hline & 200 & & & & & \\
\hline
\end{tabular}

Table 4. $P M_{10}$ and adherent specific pollutants in 2020

\begin{tabular}{|l|l|l|l|l|l|l|}
\hline Parameters & $\mathbf{P M}_{\mathbf{1 0}}$ & $\mathbf{P b}$ & $\mathbf{A s}$ & $\mathbf{C d}$ & $\mathbf{N i}$ & $\mathbf{B}(\mathbf{a}) \mathbf{P}$ \\
\hline Facilities & \multicolumn{2}{|c|}{$\boldsymbol{\mu g} / \mathbf{m}^{\mathbf{3}}$} & \multicolumn{4}{|c|}{$\mathbf{n g} / \mathbf{m}^{\mathbf{3}}$} \\
\hline $\begin{array}{l}\text { „Nikola } \\
\text { Tesla” } \\
\text { Rakovica P.S. }\end{array}$ & $\mathbf{4 2 . 3 3}$ & 0.022 & 1.82 & 1.57 & 5.85 & $\mathbf{3 . 4 5}$ \\
\hline $\begin{array}{l}\text { „M. } \\
\text { Jovanovič } \\
\text { Kragujevac } \\
\text { P.S., }\end{array}$ & 36.32 & 0.41 & 1.76 & 0.63 & 6.38 & \\
\hline $\begin{array}{l}\text { „Sveti Sava“, } \\
\text { Niš P.S. }\end{array}$ & $\mathbf{4 8 . 1 0}$ & 0.004 & 1.42 & 1.83 & 1.92 & \\
\hline $\begin{array}{l}\text { „Đura Jakšic“, } \\
\text { Kać P.S. }\end{array}$ & 27.72 & 0.006 & 0.82 & 0.41 & 3.84 & $\mathbf{2 . 4 2}$ \\
\hline $\begin{array}{l}\text { S.Kamenica, } \\
\text { SOS „Dečije } \\
\text { selo“* }\end{array}$ & 22.86 & 0.005 & 076 & 0.26 & 4.11 & $\mathbf{1 . 0 9}$ \\
\hline $\begin{array}{l}\text { Annual limit } \\
\text { values }\end{array}$ & $\mathbf{4 0}$ & $\mathbf{0 . 5}$ & $\mathbf{6}$ & $\mathbf{5}$ & $\mathbf{2 0}$ & $\mathbf{1}$ \\
\hline
\end{tabular}

*SOS Children`s village

\section{DISCUSSION}

In most cases educational facilities are located at important city intersections, which is justified by choosing traffic-induced pollutants, such as $\mathrm{SO}_{2}, \mathrm{NO}_{2}$ and soot to be measured on daily basis at 13 out of 27 facilities $[15,16,17]$. On the other hand there is a significant disparity between the need to monitor the exposure of children to extremely high values of $\mathrm{SO}_{2}$ $[18,19]$ and unnecessary monitoring of atmospheric deposition, in which increased values of any parameter are regularly not recorded [14].

Through more rational allocation of resources, some better distribution of measuring stations and determining of parameters to monitor according to continuous air pollution sources a more valuable assessment of the exposure to air pollution of vulnerable population groups (children from preschool to adolescence age), could be made. For example, the town of Bor and Krivelj village have a long history of environmental pollution due to copper mining and smelting activities, and AQ monitoring is covered with a substantial number of stations and parameters [20, 21].

But, besides being an industrial town, we have children living there, attending institutions of all levels of education (5 primary schools, kindergartens, a high school and University of Technology in Bor) in which they are exposed to pollutans entering from outdoor to indoor space, being well documented through literature [22, 23]. Results of the APHEKOM network study indicate that near-road-related pollution may be responsible for a significant portion of the burden of asthma in children, as well as chronic heart diseases, in adults [23].

Particularly in Bor, more emphasis should be put on arsenic and cadmium concentraions adherent to PM10 particles, due to high mobility of airborne particles from outdoor to indoor environment $[24,25]$.

\section{CONCLUSIONS}

After having an insight into the frequency and choice of monitoring air quality at the local level in Serbia, a conclusion is imposed on an existing strong need to make indicator corrections in AQ monitoring in industrially contaminated urban settlements in Serbia and allocate financial and technical resources for continuous monitoring in front of more schools and kindergartens in Bor, Smederevo, Pančevo, Šabac, as well as determining parameters depending on the source of emissions [14,26-29].

\section{REFERENCES}

[1] Wolfe MK, McDonald NC, Arunachalam S, Baldauf $\mathrm{R}$, Valencia A. Impact of school location on children's air pollution exposure, Journal of Urban Affairs, 17 p., 2020,

[2] INCA. INCA Comparative Tables (Online). Interational Review of Curriculum and Assessment Framework Internet Archive (INCA), London, UK, 2009. 
[3] Laumbach RJ, Kipen HM. Respiratory health effects of air pollution: Update on biomass smoke and traffic pollution, J Allergy Clin Immunol, 129:3-11, 2012.

[4] Janssen NAH, Brunekreef B, Van Vliet P, Aarts F, Meliefste K, Harssema H, Fischer, P. The Relationship between Air Pollution from Heavy Traffic and Allergic Sensitization: Bronchial Hyperresponsiveness, and Respiratory Symptoms in Dutch School Children. Environ. Health Perspect. 111: 15121518,2003

[5] Hoek G, Brunekreef B, Goldbohm S, Fischer P, van den Brandt PA. Association between mortality and indicators of traffic-related air pollution in the Netherlands: a cohort study, The Lancet. 360: 12031209, 2002.

[6] Dales R, Wheeler A, Mahmud M, Frescura AM, Smith-Doiron M, Nethery E, Liu L. The Influence of Living near Roadways on Spirometry and Exhaled Nitric Oxide in Elementary Schoolchildren, Environ. Health Perspect. 116: 1423-1427, 2008.

[7] Chen T. M, Gokhale J, Shofer S, Kuschner WG. Outdoor air pollution: nitrogen dioxide, sulfur dioxide, and carbon monoxide health effects, $\mathrm{Am} \mathrm{J} \mathrm{Med}$ Sci.,333(4):249-56, Apr 2007.

[8] Hwang BF, Lee YL, Lin YC, Jaakkola JJK, .Guo YL. Traffic related air pollution as a determinant of asthma among Taiwanese school children, Thorax, 60: 467-473,2005.

[9] Schwartz J. Air Pollution and Children's Health, $P e$ diatrics, 113:1037-1043, 2004.

[10]Smith K. R. Indoor air pollution in developing countries: recommendations for research. Indoor Air, 12:1-7, 2002.

[11]Bruce N, Perez-Padilla R, Albalak R. (2000) Indoor air pollution in developing countries: a major environmental and public health challenge, Bull World Health Organ, 78(9): 1078-1092, 2000.

[12]Kumie A, Emmelin A, Wahlberg S, Berhane Y, Ali A, Mekonnen E, Brandstrom D, Magnitude of indoor $\mathrm{NO}_{2}$ from biomass fuels in rural settings of Ethiopia, Indoor Air, 19(1):14-21, 2009.

[13]Vujić B. B, et al. Air Quality Monitoring and Modeling Near Coal Fired Power Plant, THERMAL SCIENCE, , Vol. 23, No. 6B, pp. 4055-4065, 2019.

[14]https://www.batut.org.rs/index.php?content=1413

[15]Health effects of transport-related air pollution ISBN 92-890-1373-7 World Health Organization 2005 [16]https://www.euro.who.int/_data/assets/pdf_file/000 6/74715/E86650.pdf

[17]Fuller G, Anderson R, Atkinson R, Mills IC, Walton $H$. Traffic-related pollution and asthma prevalence in children. Quantification of associations with nitrogen dioxide. Air quality atmosphere and health, 2014.

[18]Simonovski A, Tasić V, Apostolovski-Trijić T, Milikić N, Božilov A. ACTA UNIVERSITATIS Series: Working and Living Environmental Protection Vol. 17, No 2, pp. 131 - 137, 2020, https://-doi.org/10.22190/FUWLEP2002131S

[19]Nikolić Đ, Milošević N, Mihajlović I, Živković Ž, Tasić V, Kovačević R, Petrović N. Multi-criteria Analysis of Air Pollution with $\mathrm{SO}_{2}$ and $\mathrm{PM}_{10}$ in Urban Area Around the Copper Smelter in Bor, Serbia, Water, Air and Soil Pollution, 206(1): 369-383, 2010.

[20]Stanojević A. B. Air pollution emission from the copper smelter Complex Bor in Serbia. Ann Environ Sci Toxicol, 5 (1): 023-026, 2021.

[21]Tasić V, Milošević N, Kovačević R, Jovašević-Stojanović M, Dimitrijević M. Indicative levels of PM in the ambient air in the surrounding villages of the copper smelter complex Bor, Serbia, Chemical Industry \& Chemical Engineering Quarterly, 18 (4) 643-652, 2012.

[22]Janssen NAH, van Vliet PHN, Aarts F, Harssema H, Brunekreef B. Assessment of exposure to traffic related air pollution of children attending schools near motorways. Atmospheric Environment, 35:38753884, 2001.

[23]Perez L, Declercq Ch, Iňiguez C, Aguilera I, Badaloni Ch, Ballester F, Bouland C, Chanel O, Cirarda F. B, Forastiere F, Forsberg B, Haluza D, Hedlund B, Cambra K, Lacasaňa M, Moshammer H, Otorepec P, Rodrguez-Barranco M, Medina S, Künzli N, Chronic burden of nearroadway traffic pollution in 10 European cities (APHEKOM net-work), Eur Respir J, 42: 594-605, 2013.

[24]Martin R, Dowling K, Pearce D, Sillitoe J, Florentine S. Health Effects Associated with Inhalation of Airborne Arsenic Arising from Mining Operations, Geosciences, 4(3), 128-175, 2014, https://doi.org/10.3390/geosciences4030128

[25]Kovačević R, Jovašević-Stojanović M, Tasić V, Milošević N, Petrović N, Stanković S, Matić Besarabić S. PRELIMINARY ANALYSIS OF LEVELS OF ARSENIC AND OTHER METALIC ELEMENTS IN PM10 SAMPLED NEAR COPPER SMELTER BOR (SERBIA), Chemical Industry \& Chemical Engineering Quarterly, 16 (3) 269-279, 2010. 
[26]Olufemi AC, Mji A, Mukhola MS. Health risks of exposure to air pollutants among students in schools in the vicinities of coal mines, Energy Exploration \& Exploitation, Vol. 37(6) 1638-1656, 2019, https://doi.org/10.1177\%2F0144598718765489

[27]Bergstra AD, Brunekreef B, Burdorf A. The effect of industry-related air pollution on lung function and respiratory symptoms in school children. Environ Health 17, 30, 2018, https://doi.org/10.1186/s12940018-0373-2
[28]Rovira E, Cuadras A, Aguilar X, Esteban L, BorràsSantos A, Zock Jan-Paul, Sunyer J. Asthma, respiratory symptoms and lung function in children living near a petrochemical site, Environmental Research, Vol. 133, pp. 156-163, 2014, ISSN 0013-9351, https://doi.org/10.1016/j.envres.2014.05.022.

[29]Matić B. I, Rakić U. D, Dejanović SM, Jovanović V. S, Jevtić M. R, Đonović NŽ. Industrially Contaminated Areas in Serbia as a Potential Public Health Threat to the Exposed Population, Tehnika, 72(3):441-7, 2017.

\section{REZIME PRAĆENJE KVALITETA URBANOG AMBIJENTALNOG VAZDUHA NA LOKACIJAMA
PREDŠKOLSKIH I ŠKOLSKIH USTANOVA U SRBIJI}

Izlaganje dece polutantima iz životne sredine tokom perioda značajnih za njihov fiziološki razvoj može dovesti do dugotrajnih zdravstvenih efekata, disfunkcije i bolesti. Cilj ovog rada bio je da se usredsredi na relativno oskudnu praksu praćenja kvaliteta ambijentalnog vazduha na lokacijama predškolskih $i$ školskih ustanova u Srbiji, u okviru lokalne mreže stanica za praćenje KV. Ovo je bila studija preseka, obuhvatajući period od 2015. do 2020. godine. Merna mesta ( $\mathrm{SO}_{2}, \mathrm{NO}_{2}$, čađ, PM $\left.\mathrm{M}_{10}\right)$ locirana su ispred obrazovnih ustanova (osnovne škole, vrtići, srednja škola) u urbanim celinama. Rezultati potiču iz kontinuiranog programa koji sprovodi Institut za javno zdravlje Srbije. Samo su vrednosti PM 10 i B(a)P bile iznad srednje godišnje GV ispred izabranih objekata u 2020. godini. Apsolutni broj mernih mesta za monitoring urbanog ambijentalnog vazduha od strane lokalne mreže ZJZ, kao i njihov broj ispred obrazovnih objekata su u ovom periodu porasle sa 73/17 na 172/26. U procentualnom pogledu, došlo je do pada sa 23,29 na 15,12\% u odnosu na sva merna mesta iz izveštaja. Kako se takvi objekti uglavnom nalaze na važnim gradskim raskrsnicama, u cilju procene izloženost zagađenom vazduhu ugroženih grupa stanovništva (deca od predškolskog do adolescentskog uzrasta), postoji potreba za lociranjem mernih mesta u njihovoj blizini.

Ključne reči: obrazovne ustanove, zagađenje ambijentalnog vazduha, merna mesta 